\title{
Perceptual completion and object-based representations in short-term visual memory
}

\author{
PETER WALKER and SIMON J. DAVIES \\ University of Lancaster, Lancaster, England
}

\begin{abstract}
Object-based representations in visual short-term memory (VSTM) were examined using a change detection memory task. A display comprising two rows of four differently colored elements was followed by a probe display in which only one of the rows reappeared. On same trials, the probed row was identical to the corresponding row in the memory display. On different trials, two of the elements in the probed row had their colors exchanged. In each memory display, a task-irrelevant visual element appeared between the two rows, with the potential to function as an occluder. Performance was enhanced when perceptual completion meant that four, rather than eight, objects were perceived in the memory display and when the probe display revealed that the occluded elements continued behind the occluder. It appears that several forms of representation can co-occur to support VSTM, one of which is object based.
\end{abstract}

How an object's visual feature conjunctions are perceived has been investigated intensively since Treisman proposed herfeature integration theory (see, e.g., Treisman $\&$ Gelade, 1980). How an object's visual feature conjunctions are represented in memory is now beginning to emerge as a research theme also (see Walker \& Cuthbert, 1998, for a review). At one extreme, Treisman, Sykes, and Gelade (1977) suggested that the linking of an object's visual features might be contingent on the physical presence of the object, so that when the object is no longer in view the "glue" that kept its features together dissolves and the features become dissociated. The work of Magnussen and his colleagues reinforced the view that separate feature-specific modules can support visual short-term memory (VSTM) performance (see Magnussen \& Greenlee, 1999, for a review). Other research also has indicated that feature conjunctions can be inadequately represented, if represented at all, in VSTM (Light \& Berger, 1976; Stefurak \& Boynton, 1986; Treisman, 1977; Treisman et al., 1977).

In contrast, Walker and Cuthbert (1998) exploit the unitization effect in memory to provide evidence that feature conjunctions can be directly represented in VSTM (see Ceraso, 1985, for an account of earlier studies of the unitization effect). In a task situation in which spatial location could not provide a link between the separate representations of an object's other features, they provided experimental evidence that feature conjunctions can be preserved in visual memory. Because this was possible only when the features were originally perceived to be-

\footnotetext{
Simon Davies is supported by a research studentship from the U.K. Economic and Social Research Council. The authors are grateful to Tania Mann for suggestions regarding the design of the displays used in the present study. Correspondence concerning this article should be addressed to P. Walker, Department of Psychology, Lancaster University, Lancaster LA1 4YF, England (e-mail: p.walker@lancaster.ac.uk).
}

long to the same object (i.e., when the shapes themselves were colored, as opposed to the backgrounds against which they appeared), Walker and Cuthbert argued that memory for the conjunctions was object based. In a similar vein, Irwin and Andrews (1996) examined the contingencies in the recall of different features from multi-item displays and argued that object-based representations, possibly in the form of object files (see Kahneman, Treisman, \& Gibbs, 1992), can bridge the brief time interval separating successive eye fixations.

Luck and Vogel (1997) and Vogel, Woodman, and Luck (2001) also claimed that VSTM representations can be object based. They based this claim on their demonstration that the overall level of performance in a VSTM task appears not to be determined by the number of feature values needing to be retained but, instead, by the number of objects that these values map onto. For example, memory for a display was just as good when the display comprised four double-feature items (e.g., four bars distinguished according to both their orientation and their color) as when it comprised four single-feature items (e.g., four bars distinguished only by their color).

Wheeler and Treisman (2002) reiterated a point also made by Luck and Vogel (1997) themselves that all but one of their results could be explained by assuming that the values in each feature domain (e.g., the different colors in a display) are preserved in separate feature-specific modules, or what Treisman referred to as separate feature maps (see, e.g., Treisman, 1998). If different featurespecific modules have comparable limited capacities and a feature value needs to be attended to be preserved in a module, the misleading impression could be gained that VSTM is object based. The values preserved in each feature module would be just those pertaining to the subset of objects to which the participants had attended.

Luck and Vogel (1997) pointed out, however, that this alternative to the object-based explanation cannot accom- 
modate their observation that memory performance was not affected by bestowing each object in a display with two values from the same feature domain. Thus, when the squares in a display were bicolored, rather than singly colored, memory for the display continued to be determined by the number of squares (objects) and was uninfluenced by the fact that the number of colors in the display had been doubled. This is a critical observation because, if performance is mediated by a set of feature-specific modules that have limited capacity, doubling the number of feature values to be retained in each module should increase the likelihood that the capacity of the modules is exceeded. However, Wheeler and Treisman (2002) were unable to replicate this important observation and attributed their failure to the fact that the colors used by Luck and Vogel were so bright that the two colors assigned to the same square blended into a single color. Schneider, Deubel, and Wesenick (2000) have also failed to replicate the finding reported by Luck and his colleagues.

To examine matters further, Wheeler and Treisman (2002) created different versions of a change detection task that isolated short-term memory for conjunctions of features (shape-color and color-location) from memory for individual features (color, shape, and location). Of course, representations of feature conjunctions that are separate from representations of individual features would appear to qualify as object-based representations, especially when a shape-color conjunction is represented directly, without any reference to spatial location. By contrasting performance with single-item versus multipleitem test displays, Wheeler and Treisman dissociated memory for feature conjunctions from memory for individual features, indicating that at least some, but not necessarily all, aspects of VSTM are object based. This interpretation is most compelling with regard to their investigation of shape-color conjunctions, where they took steps to ensure that performance was not supported by space-based representations (i.e., by making spatial location an irrelevant feature).

The present study adopted a complementary experimental strategy to that employed by Luck and Vogel (1997) to confirm that some representations in VSTM can be object based. A task situation was created in which the to-be-remembered feature values in a display were held constant while the number of perceived objects these features mapped onto was varied. If VSTM for the same display could be shown to vary with the number of perceived objects, we would have strong evidence for a contribution from object-based memory representations. To demonstrate this, we decided to capitalize on the phenomenon of perceptual completion. Apart from being a means whereby the number of perceived objects in a display can be varied without changing the to-be-remembered features, perceptual completion is a phenomenon that itself speaks to the object-based nature of perceptual representations (e.g., Kanizsa, 1979; Kanizsa $\&$ Gerbino, 1982). It does so because it represents what Rock (1983) referred to as the constancy mode of per- ceiving, whereby perception reflects the nature of an object, rather than just what is directly visible to the perceiver from a particular vantage point.

\section{EXPERIMENT 1}

Consider the memory display illustrated in Figure 1A, in which identically shaped elements appearing in various colors are organized as two vertically aligned rows. As in the displays created by Luck and Vogel (1997), repetition of colors is allowed. However, there are various constraints regarding the placement of the colorsnamely, that no color is repeated within a row, the same colors appear in each row, and no duplicated colors are vertically aligned. Apart from the regular spatial organization of the elements and the constraints with regard to the distribution of the colors, such a display is, in principle, equivalent to those used in some of Luck and Vogel's experiments in which memory for colors in their locations was examined. Hence, if short-term memory for the locations occupied by the colors were to be examined with displays such as the one illustrated in Figure 1A, we would expect to observe similar results to those reported by Luck and Vogel. Participants could be asked, for example, to indicate whether a test display is identical to a preceding memory display. When they differ, the change could involve an exchange of positions by two colors from the same row, but not so that the two instances of a particular color become vertically aligned (compare, e.g., Figures 1A and 1B). The participants would not be able to predict in which row the color switch was to occur. Because a change involves two colors' exchanging positions, it could not be detected simply on the basis of the set of color values present in the display (i.e., the participants could not check a list of colors present). Instead, the participants would need to respond on the basis of their memory for the colors in their locations. In view of the assumed limited capacity of VSTM, the participants would not be expected to perform without error.

Adding an irrelevant item to the displays should not alter the pattern of performance. Consider Figures 1C and 1D, for example. Here, a cylinder has been positioned between the two rows of colored elements. Although the vertical alignment of the elements in the two rows is now made more salient, there is no obvious reason why this should have an impact on performance. Although the shapes and positions of the pairs of vertically aligned elements make them relatable and, therefore, candidates for perceptual completion (see Kellman \& Shipley, 1991), the differences in color discourage completion from occurring. Obviously, the potential for perceptual completion could be enhanced by allowing instances of the same color to be vertically aligned. However, if the duplicated colors were all vertically aligned in the display, not only would perceptual completion be strong, but also detecting change could be based on memory for just one of the rows of colored elements. Hence, it would not be easy to show that improved change detection performance came 


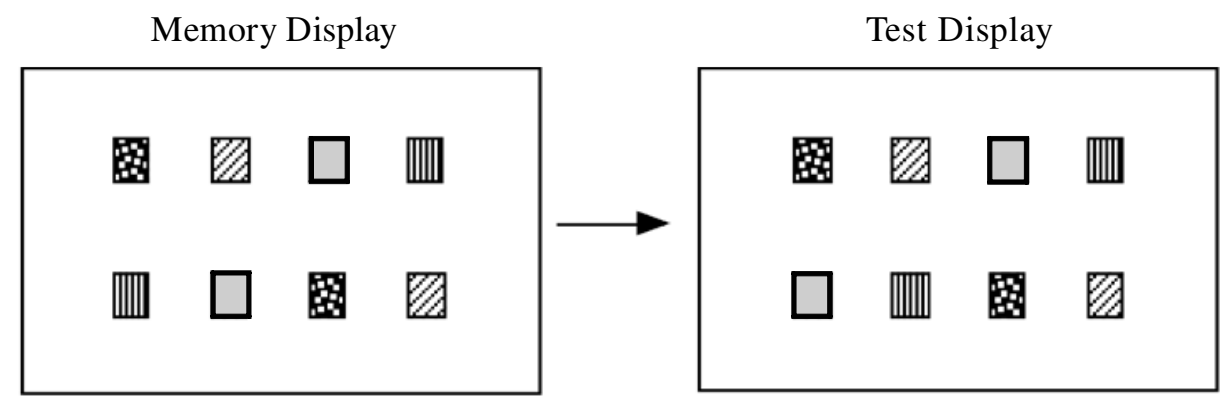

A

B

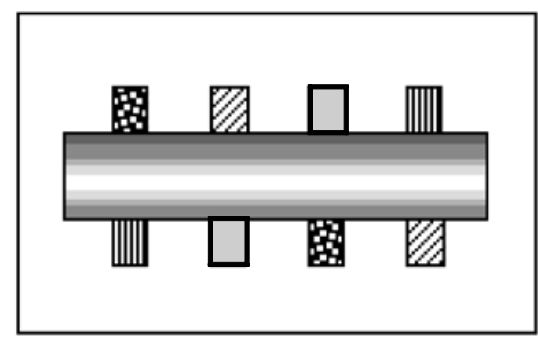

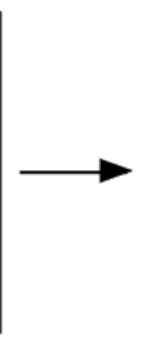

$\mathrm{C}$

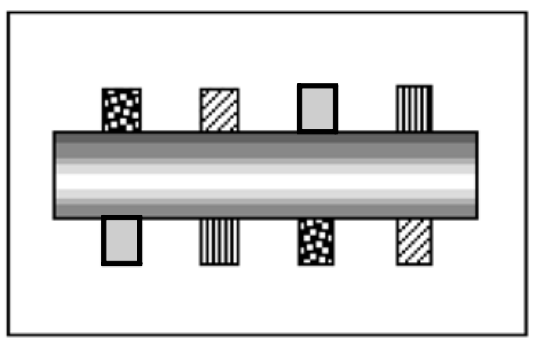

$\mathrm{D}$

Figure 1. Two versions of displays that could be used to investigate visual short-term memory for colors in their locations. Participants are required to indicate whether a test display differs from the preceding memory display in terms of the placement of the colors. When a change occurs, it involves an exchange of positions by two colors from a row, although not in a way that would mean that the two instances of a color become vertically aligned. The two test displays, $B$ and $D$, are examples of trials on which a change is introduced. Despite various constraints on the spatial distribution of the elements and their colors (see the text) and the inclusion of a task-irrelevant stimulus in $C$ and $D$, the displays are equivalent to those employed by Luck and Vogel (1997) in some of their experiments. Note that in this and the other figures, color is represented through different textures.

about because perceptual completion reduced the demands of the task from remembering the colors of eight elements to remembering the colors of four objects. Instead, the participants could quickly notice that the color sequence in the two rows was identical and concentrate their efforts on remembering the colors in just one row.

To circumvent these and other practical obstacles to examining the impact of perceptual completion on change detection, the four different types of displays illustrated in Figure 2 were devised.

The orientations of the elements in the displays were varied so that completion could occur between elements occupying noncorresponding positions in the two rows. In this way, the impact of perceptual completion was dissociated from the impact of having the same sequence of colors appear in each row. In the first type of display, identically colored elements occupied adjacent positions across the two rows. Because these elements were already relatable in terms of their shape and location, perceptual completion could occur, giving the impression that four slanting bars continued behind the occluding cylinder. This type of display is labeled crossed-relatable. We expected perceptual completion to facilitate change detection by reducing the task from one in which eight col- ored elements needed to be remembered to one in which four colored objects needed to be remembered. (Note that whereas the first part of the label for each type of display refers to the spatial arrangement of the duplicated colors, the second part indicates whether the shapes and locations of the elements, quite separately from their color, can support completion by being relatable.)

To isolate the impact of perceptual completion on performance, a second type of display was created in which the ordinal positioning of the colors in the two rows remained unchanged but in which the orientations of the elements were altered to make them unrelatable and, therefore, unsupportive of completion. This type of display is labeled crossed-unrelatable (see Figure 2B). If visual memory can be supported by object-based representations and if perceptual completion reduces the number of perceived objects, we would expect change detection performance to be superior in the crossed-relatable condition, as compared with the crossed-unrelatable condition. This would be the case even though the number of colored elements in the displays and the assignment of colors to locations are equivalent in the two types of display.

A further two display types were created to check that any difference in performance between the crossed- 


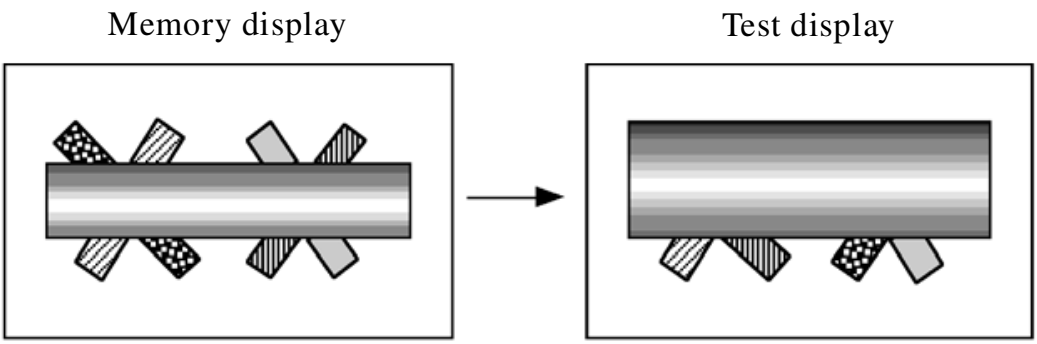

A. crossed-relatable

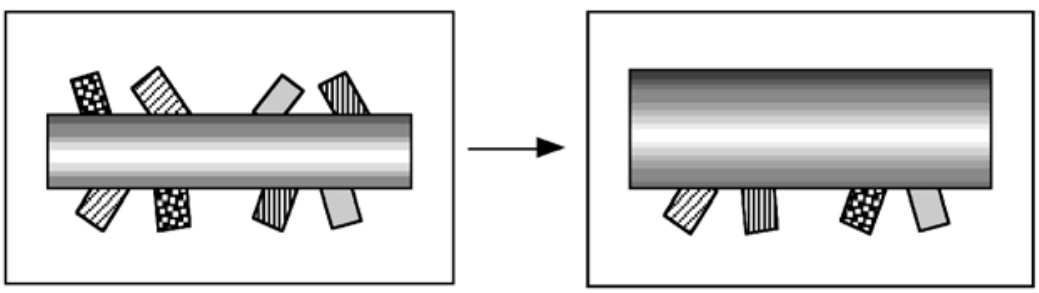

B. crossed-unrelatable

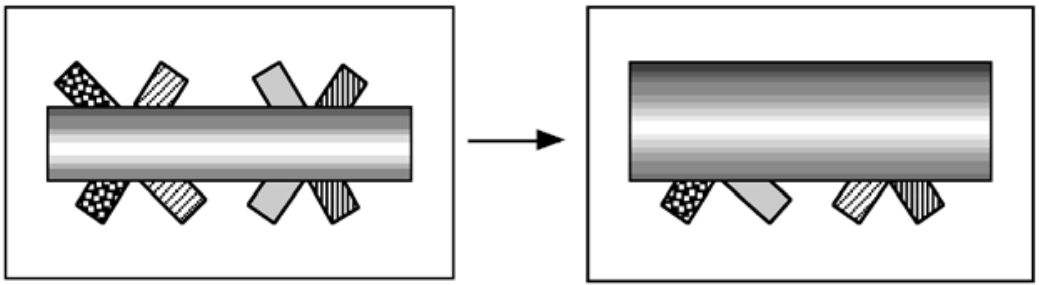

C. vertical-relatable

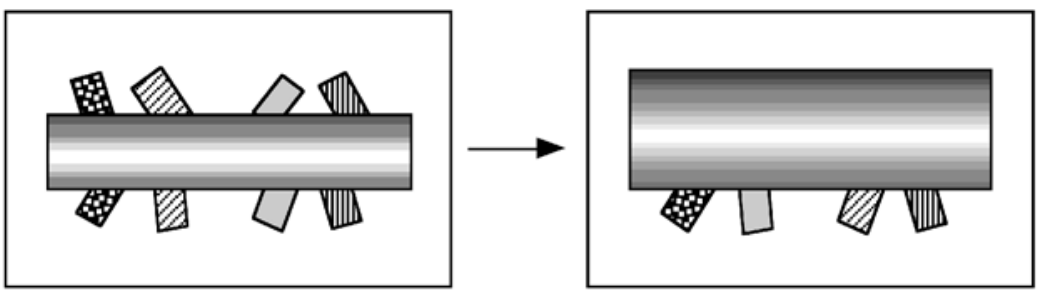

D. vertical-unrelatable

Figure 2. The four types of displays used in Experiment 1 (see the text for an explanation). Although in all of the test displays illustrated the occluder is shown to be expanded to leave only the bottom row of colored elements exposed, in practice it was equally likely to be either row that was exposed. For each type of display, a different trial is illustrated, with two colors in the bottom row exchanging positions. In this and subsequent illustrations, the experimental displays are not reproduced exactly to scale, nor is the depiction of the sizes and positions of the various components as uniform or as precise as in the experimental displays. Note that there were no borders around the colored elements in the experimental displays.

relatable and the crossed-unrelatable conditions arose from perceptual completion, and not from the relatability of the elements as determined solely by their shapes and locations. In these two additional display types, the two instances of each color were now assigned to the same ordinal position in each row (i.e., they were vertically aligned; see Figures 2C and 2D). Apart from the vertical alignment of the duplicated colors, these two display types, labeled as vertical-relatable and verticalunrelatable, were otherwise equivalent to the crossedrelatable and crossed-unrelatable displays, respectively. In terms of the relatability of adjacent items across the two rows, the contrast between the two vertical display types is identical to the contrast between the two crossed display types. In terms of the potential for perceptual completion, however, this is much more limited in the 
vertical-relatable displays than in the crossed-relatable displays (participants possibly could perceive the four bars to bend behind the occluder in the former displays).

Contrasting performance across the vertical-relatable and the vertical-unrelatable conditions was intended to confirm that it was the potential for perceptual completion in the crossed-relatable displays, rather than the relatability of the elements, that yielded levels of performance superior to those observed in the crossed-unrelatable condition. Critically, therefore, it was expected that any difference in the level of performance between the two crossed conditions would not also be observed between the two vertical conditions. It was considered possible, however, that the overall level of performance in the two vertical conditions would differ from the level observed in the two crossed conditions. For example, in the former case, participants might capitalize on the fact that the same color sequence appears in each row and concentrate their efforts on remembering the sequence in just one row. The contrast between the crossed and the vertical display types is, of course, orthogonal to the contrast between the relatable and the unrelatable versions of each display type.

We anticipated that the participants would generally scan the displays in a top-bottom direction. However, it was expected that perceptual completion would reduce the salience of the top-bottom row distinction, thereby attenuating any deficit associated with probing the bottom row. For these reasons, we expected the impact of perceptual completion to be most noticeable on trials that probed memory for the locations of the colors in the bottom row.

Finally, to ensure that performance was based on visual, rather than verbal, representations of the displays, the participants were required to undertake a concurrent verbal distractor task throughout each trial. This involved the continuous rehearsal of two randomly selected digits presented at the start of each trial, the same procedure as that employed by Luck and Vogel (1997).

\section{Method}

Participants. Twenty-four students from Lancaster University were paid $£ 4$ each for completing the experiment. All had normal or corrected-to-normal color vision and were naive as to the purpose of the study.

Although reported here as one experiment involving 24 participants, Experiment 1 comprised two separate experiments, each involving 12 participants. The second of these experiments was undertaken to see whether the results of the first could be replicated. In the event, both experiments produced the same pattern of significant results, which was revealed through their combined analysis.

Apparatus and Stimuli. Stimulus presentation and response monitoring were undertaken online using an Apple Macintosh G3 running SuperLab (V. 2.01 BetaDebug 68K). Stimuli were displayed on a color monitor with a resolution of $832 \times 624$ pixels, running at $75 \mathrm{~Hz}$ and viewed from a distance of approximately $60 \mathrm{~cm}$. The participants used the first two fingers of the preferred hand to press the $L$ and $M$ keys on the computer keyboard to register same and different decisions, respectively.

Two sets of 24 colorless templates were created. One set was used to generate the relatable displays; the other set was used to gener- ate the unrelatable displays. For the relatable displays, each of the four elongated bars measured $6 \times 42 \mathrm{~mm}$ when continued behind the occluder. For the unrelatable displays, the eight rectangular elements matched those in the relatable displays, except for their orientations and slight lateral shifts in position. The occluder in each memory display measured $89 \times 22 \mathrm{~mm}$. Each of the 24 possible arrangements of four colors (red, green, blue, and yellow) was applied to 1 template in each set. Each set of displays created in this way was then duplicated, with identical sets being used for same and different trials. A test display was created for each of the 192 memory displays. For each set of memory displays, an equal number of test displays incorporated an occluder that left either the top or the bottom row of elements visible. Which row was probed was randomly determined. This selective occlusion of one row of elements was achieved by expanding the depth of the occluder, from 22 to $37 \mathrm{~mm}$, in either an upward or a downward direction.

For a different trial, the change introduced to the test display involved two elements' exchanging their colors. There were four alternative pairings of elements in the unoccluded row that had their colors exchanged. Each of these alternatives was employed equally often. Numbering the elements from left to right as 1-4, the alternatives were 1 with 2,3 with 4,1 with 4 , and 2 with 3 . It will be noted that the elements in the displays, and especially in the crossed displays, appear to form two bicolored perceptual groups. One group incorporates the first two colors in each row; the second group incorporates the final two colors. Therefore, the various pairings of elements exchanging their colors can be distinguished on the basis of whether the colors originated from the same group of elements (1 with 2 and 3 with 4 ) or from different groups of elements ( 1 with 4 and 2 with 3 ).

The memory and test displays were spatially coincident and appeared in the center of a black outline frame measuring $10 \times 10 \mathrm{~cm}$. All of the stimuli appeared on a featureless light gray background.

Design. A completely within-subjects design was employed, with factors of trial type (same and different), display type (crossedrelatable, crossed-unrelatable, vertical-relatable, and verticalunrelatable), row (bottom and top), and change type (1 with 2, 3 with 4,1 with 4 , and 2 with 3 ). For same trials, change type was a dummy variable. The type of change assigned to each same trial was taken from the different trial that used the same memory display. The total set of trials generated by the factorial combination of these factors was completed in a random order determined afresh for each participant. Because of this, the participants were not able to anticipate the nature of each trial and could not adopt different strategies for different types of trials.

Procedure. The participants initiated the sequence of events on each trial by pressing the space bar on the keyboard, whereupon two randomly selected digits in the 0-9 range appeared on the screen for $500 \mathrm{msec}$. These digits were positioned at either side of the central region in which the memory and the test displays were to appear. The participants were required to repeat these two digits continuously out loud until they had responded to the forthcoming test display. The digits were replaced by a central fixation point for $750 \mathrm{msec}$. The memory display then appeared for $500 \mathrm{msec}$, surrounded by the black outline frame. An interval of $1 \mathrm{sec}$ followed, during which the occluder and the frame remained on the screen. After this, a test display with an enlarged occluder appeared until the participant responded by pressing a key to indicate whether the arrangement of the colors in the memory and the test displays was the same or different. Immediate visual feedback was provided to the participants by displaying the word correct or incorrect in the center of the screen. After responding in this way, they were allowed to stop repeating the two digits. The instructions given to the participants emphasized the need to respond accurately, rather than quickly, although it was made clear that response speed was being recorded and would be analyzed. After completing a set of 16 practice trials, each participant completed the 192 experimental trials in a random order. 
Table 1

Experiments 1 and 2: Estimates of $d^{\prime}$

\begin{tabular}{|c|c|c|c|c|}
\hline \multirow[b]{3}{*}{ Row } & \multicolumn{4}{|c|}{ Display Type } \\
\hline & \multicolumn{2}{|c|}{ Crossed } & \multicolumn{2}{|c|}{ Vertical } \\
\hline & Relatable & Unrelatable & Relatable & Unrelatable \\
\hline \multicolumn{5}{|c|}{ Experiment 1} \\
\hline Top & 1.64 & 1.63 & 1.50 & 1.69 \\
\hline Bottom & 1.38 & 0.93 & 1.33 & 1.38 \\
\hline \multicolumn{5}{|c|}{ Experiment 2} \\
\hline Top & 1.39 & 1.18 & 1.48 & 1.96 \\
\hline Bottom & 1.18 & 1.07 & 1.18 & 1.17 \\
\hline
\end{tabular}

\section{Results}

The conventional .05 probability level was adopted as the cutoff point for statistical significance. The significance levels associated with the $F$ values emerging from analysis of variance are quoted throughout. Hence, where the directions of the effects were predicted, the probability values quoted are conservative.

The results confirmed that the participants responded more accurately and more quickly on same trials than on different trials $\left[F(1,23)=144.9, M S_{\mathrm{e}}=0.04, p<.001\right.$, and $F(1,23)=4.55, M S_{\mathrm{e}}=115,622, p<.05$, for accuracy and speed, respectively] and on trials in which the top, rather than the bottom, row of elements was probed $\left[F(1,23)=15.18, M S_{\mathrm{e}}=0.01, p<.001\right.$, and $F(1,23)=$ $39.9, M S_{\mathrm{e}}=36,475, p<.001$, for accuracy and speed, respectively].

$\boldsymbol{d}^{\prime}$. Table 1 gives the values of $d^{\prime}$ for the different display types according to which row of elements was probed. The Kolmogorov-Smirnov test confirmed that the values for $d^{\prime}$ for each combination of row and condition were normally distributed, indicating that it was valid to submit them to an analysis of variance. Exactly the same pattern of results emerged when $A^{\prime}$ was used as the measure of sensitivity.

Planned contrasts failed to reveal any significant differences in sensitivity to the various types of display when the top row of elements was probed. When the bottom row of elements was probed, however, performance with vertical displays was marginally more sensitive than performance with crossed displays $[F(1,23)=3.37$, $\left.M S_{\mathrm{e}}=0.91, p=.08\right]$. In addition, whereas there was no difference in sensitivity between vertical-relatable and vertical-unrelatable displays $(F<1)$, there was a significant difference between crossed-relatable and crossedunrelatable displays $\left[F(1,23)=6.14, M S_{\mathrm{e}}=2.40, p<.025\right]$.

Accuracy. ${ }^{1}$ Table 2 gives the mean accuracy levels for each display type separately for the different levels of row and trial type. Planned contrasts confirmed that there were no significant effects of display type when the top row of elements was probed. When the bottom row of elements was probed, there was no significant difference in accuracy between the vertical and the crossed displays $\left[F(1,23)=2.25, M S_{\mathrm{e}}=0.03, p>.05\right]$ and no significant difference between the vertical-relatable and the vertical-unrelatable displays $(F<1)$. However, perfor- mance was significantly more accurate with crossedrelatable displays than with crossed-unrelatable displays $\left[F(1,23)=6.41, M S_{\mathrm{e}}=0.13, p<.025\right]$. The results from same and different trials for the bottom row were next considered separately. For same trials, the participants performed better with vertical than with crossed displays $\left[F(1,23)=6.25, M S_{\mathrm{e}}=0.46, p=.02\right]$, and no other contrasts among the display types were significant. For different trials, there was no significant difference in overall accuracy to crossed and vertical displays $(F<1)$ and no significant difference in the accuracy of responding to vertical-relatable and vertical-unrelatable displays $(F<1)$. There was, however, a significant difference in the accuracy of responding to crossed-relatable and crossedunrelatable displays $\left[F(1,23)=8.95, M S_{\mathrm{e}}=0.48, p<.01\right]$.

Finally, an analysis of the performance on different trials revealed that change type had a significant impact on accuracy whether the top or the bottom row of elements was probed $\left[F(3,69)=43.81, M S_{\mathrm{e}}=0.13, p<.00001\right.$, and $F(3,69)=42.94, M S_{\mathrm{e}}=0.16, p<.00001$, respectively]. In each case, the participants were better able to detect a change when this involved a switch between bicolored groups, rather than a switch within a bicolored group [mean accuracy $=83 \%$ and $42 \%$, respectively, for the top row, and mean accuracy $=85 \%$ and $39 \%$, respectively, for the bottom row; $F(1,23)=99.63, M S_{\mathrm{e}}=16.53$, $p<.00001$, and $F(1,23)=85.00, M S_{\mathrm{e}}=20.18, p<.00001$, for the top and the bottom rows, respectively].

Correct response times. Response times (RTs) for each individual participant were screened to identify those associated with an incorrect response, plus those associated with a correct response, that were excessively slow (i.e., greater than $2 S D$ s above the participant's mean correct RT). Prior to analysis, any excessively slow RTs were replaced by the participant's cutoff value, although simply removing them led to the same conclusions. Some cells in the design were empty because they were associated with incorrect responses only.

Table 2

Experiments 1 and 2: Mean Accuracy Levels (as Percentages)

\begin{tabular}{|c|c|c|c|c|c|}
\hline \multirow[b]{3}{*}{ Row } & \multirow{3}{*}{$\begin{array}{l}\text { Trial } \\
\text { Type }\end{array}$} & \multicolumn{4}{|c|}{ Display Type } \\
\hline & & \multicolumn{2}{|c|}{ Crossed } & \multicolumn{2}{|c|}{ Vertical } \\
\hline & & Relatable & Unrelatable & Relatable & Unrelatable \\
\hline \multicolumn{6}{|c|}{ Experiment 1} \\
\hline Top & same & 91 & 91 & 91 & 92 \\
\hline & different & 64 & 62 & 59 & 65 \\
\hline Mean & & 78 & 77 & 75 & 78 \\
\hline Bottom & same & 81 & 76 & 85 & 87 \\
\hline & different & 68 & 58 & 62 & 61 \\
\hline Mean & & 75 & 67 & 73 & 74 \\
\hline \multicolumn{6}{|c|}{ Experiment 2} \\
\hline Top & same & 86 & 83 & 92 & 94 \\
\hline & different & 63 & 59 & 55 & 71 \\
\hline Mean & & 74 & 71 & 74 & 83 \\
\hline Bottom & same & 78 & 76 & 83 & 88 \\
\hline & different & 63 & 66 & 59 & 52 \\
\hline Mean & & 71 & 71 & 71 & 70 \\
\hline
\end{tabular}


Table 3

Experiments 1 and 2: Mean Correct Response Times (in Milliseconds)

\begin{tabular}{|c|c|c|c|c|}
\hline \multirow[b]{3}{*}{ Trial Type } & \multicolumn{4}{|c|}{ Display Type } \\
\hline & \multicolumn{2}{|c|}{ Crossed } & \multicolumn{2}{|c|}{ Vertical } \\
\hline & Relatable & Unrelatable & Relatable & Unrelatable \\
\hline \multicolumn{5}{|c|}{ Experiment 1} \\
\hline Same & 1,257 & 1,279 & 1,189 & 1,220 \\
\hline Different & 1,328 & 1,285 & 1,269 & 1,271 \\
\hline \multicolumn{5}{|c|}{ Experiment 2} \\
\hline Same & 1,274 & 1,282 & 1,191 & 1,186 \\
\hline Different & 1,297 & 1,301 & 1,274 & 1,256 \\
\hline
\end{tabular}

Table 3 gives the mean correct RT for each display type separately for same and different trials. An analysis failed to reveal any significant effects on RT arising from perceptual completion. Thus, for both same and different trials, there was no significant difference in response speed either between the crossed-relatable and the crossedunrelatable displays or between the vertical-relatable and the vertical-unrelatable displays $(F<1$ in all cases). For same trials, RTs to the two vertical display types were significantly faster than RTs to the two crossed display types $\left[F(1,23)=9.99, M S_{\mathrm{e}}=389,035, p<.005\right]$. For different trials, the participants responded more quickly to changes that involved a color switch between bicolored groups (i.e., 1 with 4 or 2 with 3 ; mean RT $=1,184 \mathrm{msec}$ ) than to changes that involved a within-group color switch [i.e., 1 with 2 or 3 with 4; mean RT $=1,392 \mathrm{msec}$; $\left.F(1,23)=33.80, M S_{\mathrm{e}}=4,176,183, p<.00001\right]$.

\section{Discussion}

Significantly higher levels of sensitivity and accuracy were observed in response to crossed-relatable displays than in response to crossed-unrelatable displays. No equivalent differences in performance were observed between vertical-relatable and vertical-unrelatable displays, confirming that the superiority of performance with crossed-relatable displays reflected the impact of perceptual completion, rather than the relatability of the elements as determined by their shape and location. In this way, the results of Experiment 1 are consistent with the proposal that perceptual completion can enhance change detection, thereby implicating the involvement of object-based representations in VSTM.

The beneficial impact of perceptual completion on memory for the assignment of colors to shapes suggests that color can be assigned to surfaces that emerge only after structural elements in a display are interpreted as signaling surface occlusion and surface continuation. The observations of Khurana (1998) concur with this suggestion. In an investigation of the illusory assignment of colors to objects, Khurana demonstrated that the assignment of colors to structural elements can be contingent on the perceived status of the elements as belonging to an occluding or an occluded object surface.
The results from Experiment 1 also revealed two additional effects relating to other aspects of the memory displays. The first of these involved superior levels of sensitivity and accuracy in response to vertical displays than to crossed displays. This is most likely to have arisen because, with vertical displays, participants have the opportunity to reduce the task to one in which they need to remember the color sequence in just one row. In contrast to the impact of perceptual completion, this advantage for vertical displays was confined to same trials. The second additional effect involved the more accurate and quicker detection of change when this involved two elements from different bicolored groups exchanging their colors rather than two elements from the same bicolored group. This grouping effect was strong regardless of which row of elements was probed and was, by definition, confined to different trials. Like the effect being attributed to perceptual completion, this grouping effect can be seen as an instance of a more general phenomenon whereby memory for a color-location association is sensitive to the context in which the individual item appears and, more specifically, is sensitive to the spatial configuration provided by other items appearing in the same display (see, e.g., Jiang, Olson, \& Chun, 2000).

The significant impact of perceptual completion confirms that the $500 \mathrm{msec}$ for which the memory displays were presented was sufficient for some or all of the relatable items to be completed. Although previous research has indicated that the time needed for perceptual completion to occur can vary (e.g., according to the percentage of an object's surface that is occluded; see Rauschenberger \& Yantis, 2001; Shore \& Enns, 1997), it appears to take approximately $200 \mathrm{msec}$ and to occur in parallel across the visual field (Rensink \& Enns, 1998; Sekuler \& Palmer, 1992). Leaving perceptual completion aside, it also seems that a presentation time of $500 \mathrm{msec}$ is more than adequate for the encoding of eight-item displays such as the ones in the present experiments. Indeed, there are indications that extending the duration of presentation from 100 to $500 \mathrm{msec}$ makes no difference to the pattern of performance based on VSTM for multi-item displays (Jiang et al., 2000; Luck \& Vogel, 1997; Vogel et al., 2001). It is very unlikely, therefore, that any of the effects observed in Experiment 1, including those arising from perceptual completion, reflect processes involved in the encoding of the memory displays.

When estimates of the capacity of VSTM are derived using the formula advocated by Pashler (1988), the impact of perceptual completion is less than might be expected. For example, collapsing across trials that probed the top and bottom rows, capacity is estimated to be 4.86 and 4.19 elements for the crossed-relatable and the crossed-unrelatable displays, respectively. Given that the capacity of VSTM has previously been estimated to be approximately three to four items and given the argument that perceptual completion enhanced performance by reducing the number of items to be remembered from 
eight to four, capacity in the crossed-relatable condition might be expected to be double that observed in the crossed-unrelatable condition. ${ }^{2}$ Instead, we see a rather modest $16 \%$ increase.

The results demonstrate, however, that a number of factors other than perceptual completion had an impact on performance, implying that there were several bases on which the participants were detecting change. There is no reason, for example, why a visual representation of completed objects should replace the original representation of eight separate elements. Instead, the former could supplement the original representation, giving participants the option of referring to either form of representation to detect change. Indeed, the results revealed a strong tendency for the participants to code the top row of four elements first, separately from other aspects of the memory displays. This meant that the impact of perceptual completion was confined to those occasions upon which memory for the bottom row was probed. When this is taken into account and the capacity of VSTM is derived from performance in relation to the bottom row only, estimates of 4.85 and 3.60 are obtained for the crossed-relatable and the crossed-unrelatable displays, respectively, representing a $35 \%$ gain from perceptual completion. This compares with a $22 \%$ gain for the vertical-unrelatable (capacity $=4.38$ ) over the crossed-unrelatable displays, a gain that also has been attributed to participants' being able to reduce the task to one in which the colors of only four items need to be remembered (i.e., the colors in just one row).

Extending the argument further, it was also observed that performance on different trials was superior when the change involved two colors switching between bicolored groups, rather than within a bicolored group, indicating that some further forms of perceptual organization were having an impact on performance. When the capacity of VSTM is derived from just those conditions associated with within-group switches in the bottom row, estimates of 2.76 and 1.06 are obtained for the crossed-relatable and the crossed-unrelatable displays, respectively, representing a $160 \%$ gain from perceptual completion. It appears, therefore, that it is the simultaneous impact of several representations, each concerned with a different perceptual organization of the displays, that is responsible for perceptual completion's seeming to have a smaller effect than expected. Finally, it is also worth noting that these estimates of capacity use information from both same and different trials. Given that the impact of perceptual completion was confined largely to different trials, estimates of capacity are unlikely to be the most sensitive indicator of the strength of influence from perceptual completion.

The idea that representations of different aspects of a display are not mutually exclusive of each other resonates with the findings of others (e.g., Irwin \& Andrews, 1996; Lee \& Chun, 2001; Wheeler \& Treisman, 2002). These researchers have demonstrated that feature-based and object-based visual representations co-occur and can have an impact simultaneously on VSTM performance. It is important to reiterate at this point that, whatever the evidence being provided here for object-based representations, it is not being claimed that representations in VSTM are exclusively object based.

\section{EXPERIMENT 2}

The superiority of performance with crossed-relatable displays in Experiment 1 has been attributed to the fact that perceptual completion reduced the number of to-beremembered items from eight to four. Perhaps, however, the relatable elements grouped more strongly when the conditions were such as to support perceptual completion, and it was this stronger sense of grouping, rather than perceptual completion itself, that enhanced performance. To provide converging evidence for the involvement of perceptual completion, Experiment 1 was repeated with the occluding cylinder reduced in depth so that a small gap appeared between it and the eight colored elements (see Figure 3 for an illustration of the appearance of the revised displays). Because of this gap, the sense of perceptual completion that was present in the crossedrelatable displays of Experiment 1 was now absent.

Davis and Driver (1997) and Rensink and Enns (1998) have demonstrated that perceptual completion is highly sensitive to the existence of such a gap. Indeed, Rensink and Enns argued that, for perceptual completion to occur, the relatable elements have to be separated by a completely occluded space. Crucially, however, they also argued that the perceptual grouping of elements is not disturbed by the presence of such a gap. On this account, therefore, the introduction of a gap should dissociate the effects of perceptual completion from the effects of perceptual grouping. On the assumption that the superior levels of performance in the crossed-relatable condition of Experiment 1 can be attributed to perceptual completion, rather than to an enhanced sense of perceptual grouping, it was expected that introducing the gap would eliminate the performance difference between the crossedrelatable and the crossed-unrelatable conditions.

\section{Method}

In all respects except the depth of the occluder (which was reduced from 22 to $8 \mathrm{~mm}$ ), Experiment 2 was a replication of Experiment 1 . Twelve students from Lancaster University completed Experiment 2. None had participated in Experiment 1, and all were naive as to the purpose of the study.

\section{Results}

The analysis of the results from Experiment 2 followed the same steps as those employed for Experiment 1 . It was confirmed that the participants responded more accurately, although not more quickly, on same trials than on different trials $\left[F(1,11)=115.20, M S_{\mathrm{e}}=0.02\right.$, $p<.001$, and $F(1,11)=1.82, M S_{\mathrm{e}}=62,342, p>.20$, for accuracy and speed, respectively]. The analysis also confirmed that the participants responded more accurately and more quickly on trials in which the top row, rather than the bottom row, of elements was probed $[F(1,11)=$ 


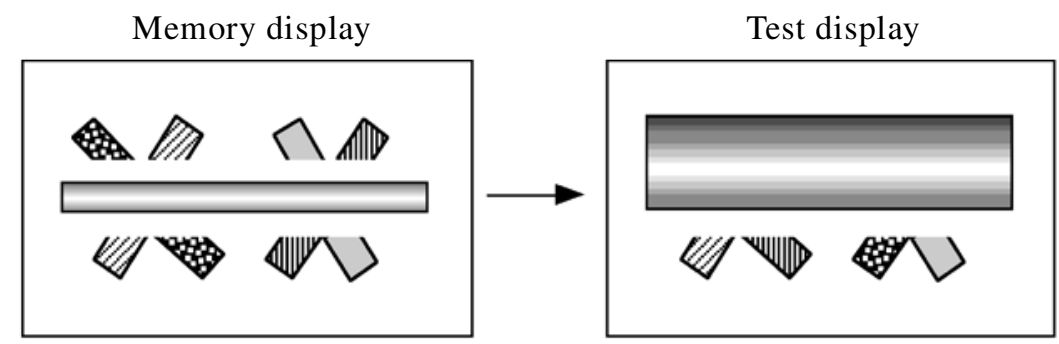

A. crossed-relatable

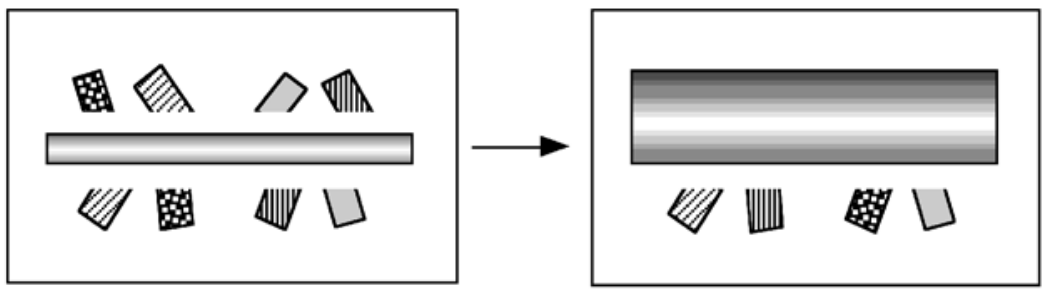

B. crossed-unrelatable

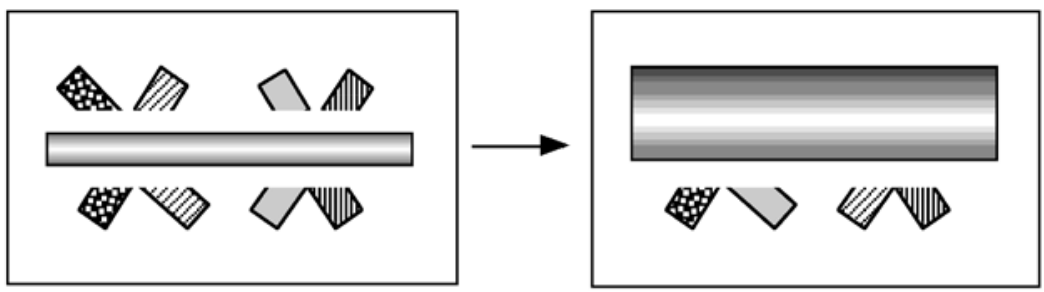

C. vertical-relatable

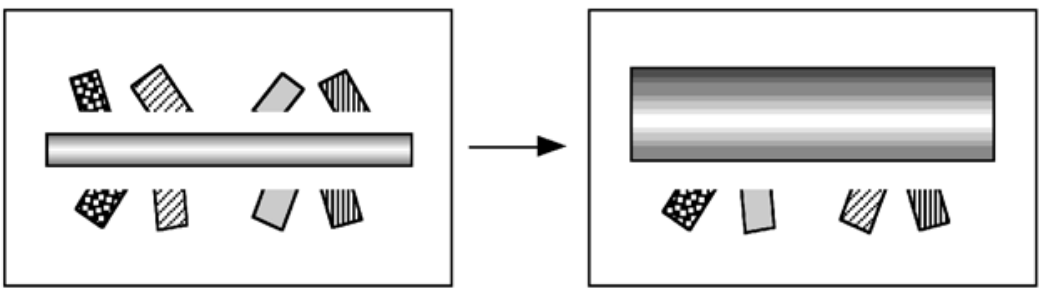

D. vertical-unrelatable

Figure 3. The four types of display used in Experiment 2. These are identical to the displays used in Experiment 1, except that the occluding cylinder is reduced in depth to leave a gap between it and the colored elements. The presence of a gap has been shown to preclude perceptual completion (see Davis \& Driver, 1997; Rensink \& Enns, 1998). Although in all of the test displays illustrated the occluder is shown to be expanded to leave only the bottom row of colored elements exposed, in practice it was equally likely to be either row that was exposed. For each type of display, a different trial is illustrated.

$11.20, M S_{\mathrm{e}}=0.01, p<.01$, and $F(1,11)=15.41, M S_{\mathrm{e}}=$ $60,101, p<.005$, for accuracy and speed, respectively].

$\boldsymbol{d}^{\prime}$. Table 1 gives the values of $d^{\prime}$ for each display type according to which row of elements was probed. When the top row of elements was probed, performance on vertical displays was more sensitive than performance on crossed displays $\left[F(1,11)=6.79, M S_{\mathrm{e}}=2.28, p<.05\right]$. Crucially, there was no significant difference in sensitivity to the crossed-relatable and the crossed-unrelatable displays $\left[F(1,11)=1.19, M S_{\mathrm{e}}=0.26, p>.10\right]$, although the participants were significantly more sensitive when responding to vertical-unrelatable displays than to verticalrelatable displays $\left[F(1,11)=14.45, M S_{\mathrm{e}}=1.38, p<.01\right]$. Planned contrasts failed to reveal any significant variation in sensitivity across the different types of display when memory for the bottom row of elements was probed (all $\left.F_{\mathrm{s}}<1\right)$.

Accuracy. Table 2 gives the mean accuracy levels for each display type separately for the different levels of row and trial type. For same trials, performance was su- 
perior with vertical displays than with crossed displays, although this effect was only marginally significant when the bottom row was probed $\left[F(1,11)=7.08, M S_{\mathrm{e}}=\right.$ $0.36, p<.025$, and $F(1,11)=3.43, M S_{\mathrm{e}}=0.36, p=.09$, for the top and the bottom rows, respectively]. No other contrasts among display types approached significance. For different trials, there was no difference in accuracy either between vertical and crossed displays in general or between crossed-relatable and crossed-unrelatable displays $(F<1$ in both cases). Post hoc tests suggested that performance with the vertical-unrelatable displays was exceptional, although in contrasting ways, depending on which row of elements was probed. This unexpected result is difficult to interpret and, until it is replicated, is regarded as anomalous.

Change type had a significant impact on accuracy whether the top or the bottom row of elements was probed. In each case, the participants were better able to detect a change when this involved a color switch between bicolored groups rather than a switch within a group [mean accuracy $=83 \%$ and $42 \%$, respectively, for the top row, and mean accuracy $=79 \%$ and $41 \%$, respectively, for the bottom row; $F(1,11)=63.29, M S_{\mathrm{e}}=8.06, p<.00001$, and $F(1,23)=104.79, M S_{\mathrm{e}}=6.75, p<.00001$, for the top and bottom rows, respectively].

Correct RTs. Table 3 gives the mean correct RT for each display type separately for same and different trials. For same trials, the only significant effect to emerge confirmed that RTs to the two vertical display types were faster than those to the two crossed display types $\left[F(1,11)=7.93, M S_{\mathrm{e}}=381,797, p<.02\right]$. For different trials, there were no effects of display type (all $F_{\mathrm{S}}<1$ ). However, the effect of change type was highly significant, once again revealing that the participants were quicker to detect a change that involved a between-group color switch (mean RT = 1,201 msec), rather than a withingroup color switch [mean RT $=1,363 \mathrm{msec} ; F(1,11)=$ $\left.24.92, M S_{\mathrm{e}}=1,251,307, p<.0005\right]$.

Comparing accuracy across Experiments 1 and 2. When the levels of accuracy achieved in those conditions associated with perceptual completion (i.e., on different trials in which the bottom row of elements was probed) were compared directly across Experiments 1 and 2, the difference between the crossed-relatable and the crossedunrelatable conditions proved to be significantly greater in the first experiment $\left[F(1,34)=5.01, M S_{\mathrm{e}}=0.06, p<.05\right]$.

\section{Discussion}

In Experiment 2, a small gap was introduced between the colored elements and the occluder to preclude perceptual completion but leave the opportunity for perceptual grouping undisturbed (see Davis \& Driver, 1997; Rensink $\&$ Enns, 1998). As was predicted, the participants ceased responding differently to the crossed-relatable and the crossed-unrelatable displays. When the levels of accuracy achieved in those conditions associated with perceptual completion were compared directly across Experiments 1 and 2, the difference between the crossed-relatable and the crossed-unrelatable conditions proved to be significantly greater in the first experiment. In this way, the findings from Experiment 2 provided converging evidence that the superior levels of performance with the crossed-relatable displays in Experiment 1 specifically reflected the impact of perceptual completion, rather than an enhanced sense of perceptual grouping among the relatable elements.

Once again, the participants' sensitivity to the type of change introduced on different trials revealed a powerful effect from a form of color-based perceptual grouping. As in Experiment 1, the participants were quicker and more accurate in detecting a change when this involved two elements from different bicolored groups, rather than two elements from the same bicolored group exchanging their colors.

\section{EXPERIMENT 3}

Rensink and Enns (1998) proposed that introducing a gap between an occluder and the elements it separates leaves perceptual grouping among the elements intact but eliminates perceptual completion. This is consistent with the observation made here that whereas introducing a gap removed the performance difference between crossed-relatable and crossed-unrelatable displays, it did not remove the perceptual grouping effect reflected in the participants' sensitivity to the type of change introduced on different trials. The suggestion is, therefore, that the conditions created to induce perceptual completion were not simply giving rise to a more powerful grouping of relatable elements but, rather, were inducing a qualitatively different effect based on perceptual completion itself. To provide additional support for the involvement of perceptual completion, a further experiment was undertaken to address the issue from a different angle.

Only crossed-relatable displays were used in Experiment 3 , and the different types of memory and test displays are illustrated in Figure 4. Two types of memory display were created. The first type was identical to the crossed-relatable displays of Experiment 1, in which there was no gap between the occluder and the colored elements. These are referred to as occlusion memory displays, because the elements appear to continue and complete behind the occluder. The second type of memory display was identical to the crossed-relatable displays used in Experiment 2, in which the occluder was reduced in depth to leave a small gap between it and the colored elements, which themselves were unchanged. These are referred to as mosaic memory displays, because the elements do not appear to continue and complete behind the occluder. In the test displays, the occluder was increased in depth and displaced up or down to hide one row of colored elements. Displacing the occluder exposed a region of the display that it had previously hidden. For one type of test display, referred to as a completion test display, moving the occluder revealed more of the colored elements in a form that was consistent with their perceived 

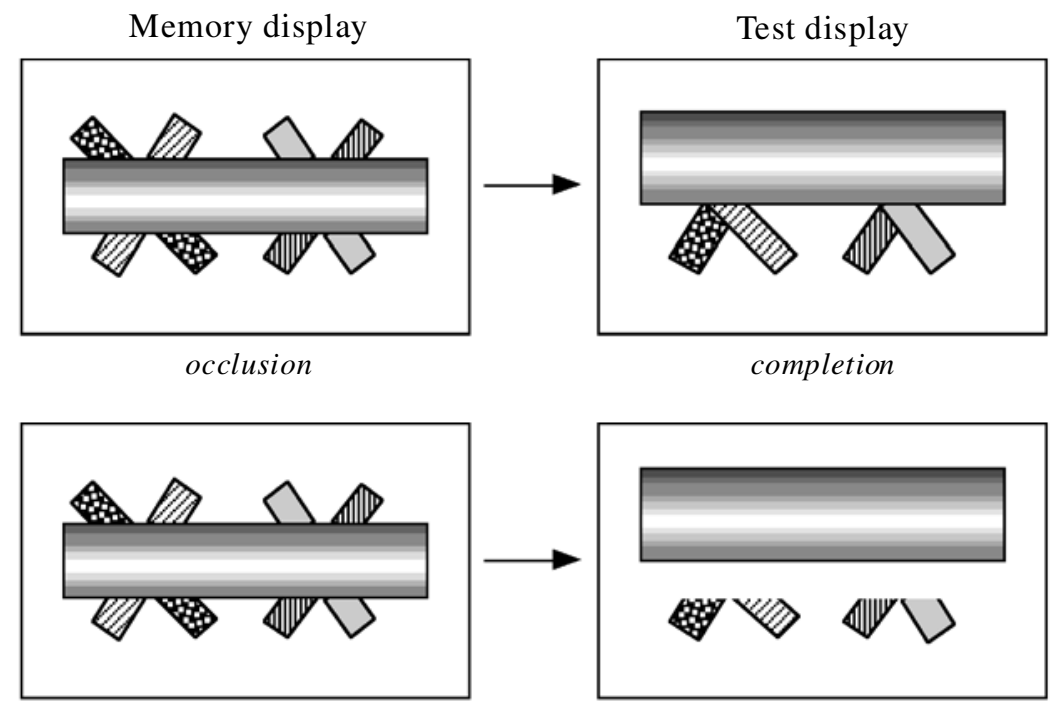

occlusion

noncompletion
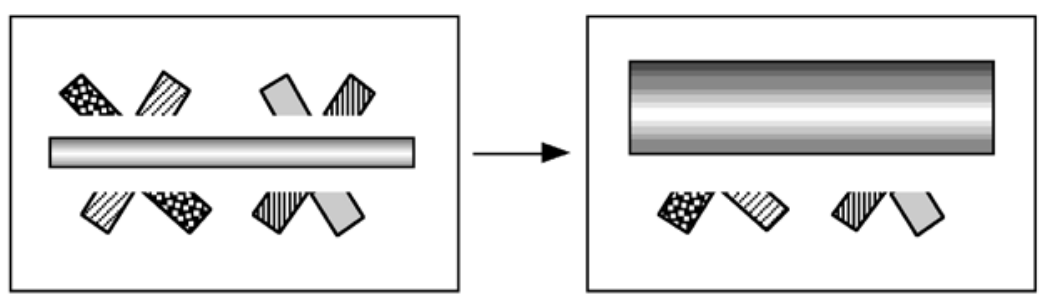

mosaic

noncompletion
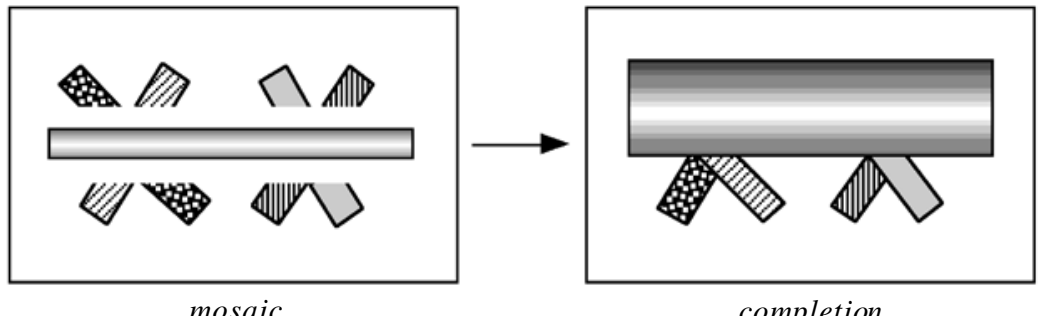

completion

Figure 4. The different types of transition between memory and test displays explored in Experiment 3. Although in all of the test displays illustrated the occluder is shown to leave only the bottom row of colored elements exposed, in practice it was equally likely to be either row that was exposed. For each transition type, a different trial is illustrated.

continuation behind the occluder in the occlusion memory displays. Because the elements were extended to a point where they still made contact with the occluder in its new position, they continued to be perceived to be partially occluded. For the other type of test display, referred to as a noncompletion test display, the colored elements remained unchanged when the occluder was moved, and the newly exposed region of the display was empty.

In Experiment 1, the impact of perceptual completion was revealed by comparing responses to crossed-relatable and crossed-unrelatable displays. Because only crossed- relatable displays were included in the present experiment, it was not possible to reveal an impact from perceptual completion in the same way. Nevertheless, it might have been expected that, on congruent trials, performance would be superior when perceptual completion reduced the number of to-be-remembered items from eight to four. In other words, the participants would be more accurate on trials involving a transition from an occlusion memory display to a completion test display than on trials involving a transition from a mosaic memory display to a noncompletion test display. In both cases, the memory and the test displays were congruent, but only in the 
former case could the two displays be compared using representations of completed objects.

Unfortunately, however, the manipulation of memorytest display congruity placed limitations on the potential utility of perceptual completion. Whereas in Experiment 1 a representation of completed elements was always useful because crossed-relatable displays were always followed by completion test displays, in the present experiment crossed-relatable memory displays that were capable of supporting completion (i.e., the occlusion memory displays) were equally likely to be followed by completion or noncompletion test displays. Consequently, the participants were not able to predict whether they would be able to use representations incorporating perceptually complete objects to compare the distribution of the colors in the memory and the test displays. It remained uncertain, therefore, whether the specific effect of perceptual completion observed on different trials in Experiment 1 would also be observed in this experiment.

Whereas a completion test display was congruent with the perceived completion of the colored elements in the occlusion memory displays, a noncompletion test display was not. Conversely, whereas a noncompletion test display was congruent with the absence of perceptual completion in the mosaic memory displays, a completion test display was not. On the understanding that perceptual completion influences the information registered in VSTM, it was predicted that performance would be superior in those conditions in which the memory and test displays were congruent (i.e., occlusion to completion and mosaic to noncompletion) rather than incongruent (i.e., occlusion to noncompletion and mosaic to completion). In other words, there would be an interaction between the nature of the memory display (whether it supported completion or not) and the nature of the test display (whether or not more of the colored elements were revealed when the occluder was displaced). Such an interaction would confirm the existence of an effect arising from perceptual completion that would be distinct from any effect that might arise from perceptual grouping. Of course, if the conditions created to induce perceptual completion facilitated performance simply by creating a stronger grouping effect among elements that remained separate, performance should be superior with the noncompletion test displays regardless of the nature of the memory display.

\section{Method}

Participants. Twenty-four students from Lancaster University were paid $£ 4$ each for completing the experiment. All had normal or corrected-to-normal color vision and were naive as to the purpose of the study.

Apparatus and Stimuli. Stimulus presentation and response monitoring were achieved in the same way as in the first two experiments. The one exception concerned a more sensitive and reliable system for recording RTs. This involved using a two-button response board that was interfaced to the computer via a National Instruments PCI-DIO-96 digital input/output card.

Two colorless display templates were prepared. One of these was used to generate the occlusion memory displays; the other was used to generate the mosaic memory displays. To ensure that the colored elements were identical in each type of memory display, the occluder was reduced slightly in depth for the mosaic displays (see Experiment 2 for details). From each template, 24 memory displays were created, one for each possible spatial arrangement of the four colors. Each of these memory displays was then duplicated to accommodate transitions to different types of test display. Each memory display was equally often followed by a completion and a noncompletion test display that probed memory for the colors in either the top or the bottom row. For each of these transition types, an equal number of trials involved a test display requiring a same or a different response. The memory and the test displays were again spatially coincident and appeared in the center of a black outline frame measuring $10 \times$ $10 \mathrm{~cm}$.

Design. A completely within-subjects design was employed, with the factors memory type (occlusion and mosaic), test type (completion and noncompletion), row (top and bottom), and trial type (same and different).

Procedure. In all essential respects, including the sequence and timing of events on each trial, the procedure was identical to that of the preceding experiments. However, the instructions now placed equal emphasis on response speed and response accuracy. After completing a set of 16 practice trials, every participant ran through a total of 192 experimental trials in a different random order. Twelve participants used the index finger on the dominant hand to respond same, and 12 used this finger to respond different.

\section{Results}

An analysis revealed that the participants did not respond differently according to which row of elements was probed $(F<1$ for both accuracy and speed). However, they did respond more accurately and more quickly on same trials than on different trials $[F(1,23)=38.70$, $M S_{\mathrm{e}}=0.10, p<.001$, and $F(1,23)=9.65, M S_{\mathrm{e}}=55,054$, $p<.005$, for accuracy and speed, respectively].

Table 4 gives the mean correct RTs for the transitions between each type of memory and test display separately for same and different trials. An analysis of RTs revealed no main effect of memory type or test type $(F<1$ in both cases). The overall interaction between these two factors was marginally significant $\left[F(1,23)=2.97, M S_{\mathrm{e}}=\right.$ $71,523, p<.10]$. When this interaction was assessed separately for same and different trials, it proved to be significant only in the former case $\left[F(1,23)=8.80, M S_{\mathrm{e}}=\right.$ $9,664, p<.01$, and $F<1$, for same and different trials, respectively].

Table 4 also gives the mean accuracy levels for the transitions between each type of memory and test display separately for same and different trials. An analysis revealed no main effect of memory type or test type and

Table 4

Experiment 3: Mean Correct Response Times (RTs, in Milliseconds) and Mean Accuracy Levels (as Percentages) Test Type

\begin{tabular}{|c|c|c|c|c|c|}
\hline \multirow{3}{*}{$\begin{array}{l}\text { Trial } \\
\text { Type }\end{array}$} & \multirow{3}{*}{$\begin{array}{c}\text { Memory } \\
\text { Type }\end{array}$} & \\
\hline & & \multicolumn{2}{|c|}{ Completion } & \multicolumn{2}{|c|}{ Noncompletion } \\
\hline & & RT & $\%$ & RT & $\%$ \\
\hline \multirow[t]{2}{*}{$\overline{\text { Same }}$} & occlusion & 1,052 & 78 & 1,070 & 83 \\
\hline & mosaic & 1,122 & 77 & 1,055 & 83 \\
\hline \multirow[t]{2}{*}{ Different } & occlusion & 1,158 & 62 & 1,183 & 57 \\
\hline & mosaic & 1,128 & 62 & 1,129 & 57 \\
\hline
\end{tabular}


no interaction between these (all $F_{\mathrm{S}}<1$ ). An inspection of Table 4 reveals that identical levels of accuracy were observed on occlusion-completion trials and on mosaicnoncompletion trials, providing unambiguous indication that perceptual completion did not improve the accuracy of performance.

Finally, it is worth noting that, in a replication of Experiment 3 in which 12 participants completed 384 trials each, the same pattern of results was obtained. Thus, for response speed only, the same congruity effect between memory type and test type was observed $(p<.01)$, and again, this was significant only for same trials $(p=.03)$.

\section{Discussion}

The results from Experiment 3 indicate that perceptual completion adds a distinct representation to VSTM. When this occurs, performance in the change detection task is enhanced when a test display reveals occluded elements to be extended in a way that is consistent with their perceived continuation behind the occluder. However, when perceptual completion is precluded by the presence of a small gap, VSTM performance is better when the elements remain unchanged in the test display. This interaction between the nature of the memory and the test displays indicates that the impact of perceptual completion does not derive from an enhanced sense of perceptual grouping among elements that, although strongly grouped, remained separate from each other.

The occluded elements were extended in the completion test displays in a way that was totally consistent with their visible appearance in the occlusion memory displays. Their shapes and surface features were preserved in these test displays (i.e., they continued as bars with the same color and plain texture). It remains for future experiments to reveal what constraints completion can place on a person's perceptual expectations regarding what will be revealed when an occluder is removed. For example, a memory-test congruity effect might not be contingent on preserving all of an occluded element's features but might arise when the occluded elements are extended in a way that preserves their shapes, but not necessarily their surface features. In other words, completion might involve perceptual inferences regarding the shapes of the emerging objects, but not their colors and textures. Alternatively, a memory-test congruity effect might arise when the occluded elements are extended in a way that preserves their surface features, but not necessarily their shapes. Finally, a memory-test congruity effect might arise when the occluded elements are extended in any way, in which case completion would be seen to induce the minimum perceptual expectation: The objects would be expected simply to continue behind the occluder, with no constraints on their visual appearance when the occluder is removed. Rensink and Enns (1998) arrived at just this conclusion on the basis of their study of perceptual completion. In the context of a primed matching task, however, Sekuler, Palmer, and Flynn (1994) reported evidence that completion induces some perceptual expectations about the shapes of the hidden portions of occluded objects. They observed that when previously occluded elements were more fully revealed in a test display, by moving the occluder, participants were quicker to respond when the extended shapes of these elements conformed to expectations based on the global shapes of the occluded elements. It is possible, therefore, that each of the alternatives just listed will find support in different task situations. Given that the occluded elements in Experiment 3 were extended in a way that preserved both their shapes and their surface features, it is not yet possible to adjudicate between these alternatives in the context of the VSTM change detection task used here.

In discussing the results of Experiment 1, it was assumed that the processes underlying same and different decisions, although overlapping, were not identical. For example, a decision to respond same might be based primarily on an early global comparison of the memory and the test displays. Although such a comparison would embrace all aspects of the task-relevant elements in the displays, it would not require localized analyses of the individual elements. However, a decision to respond different is unlikely to be a default outcome when there is insufficient evidence to justify a same decision. Rather, the decision to respond different would be reached only after additional processes are engaged to discover how the displays differ in their task-relevant features (in the present study, how the placement of the individual colors differs across the two displays). It was suggested that the impact of perceptual completion was confined to different trials in Experiment 1 because representations of completed objects were sometimes interrogated to discover the nature of any changes that had occurred.

The results from Experiment 3 differed in two ways from those gathered in Experiment 1. First, there was no enhancement of accuracy on different trials when perceptual completion was possible. It has been suggested already that the manipulation of congruity in Experiment 3 changed the contingencies between the memory and the test displays in a way that could have discouraged the participants from referring to any representations of completed objects. In addition, the instructions emphasized response speed over accuracy. Either of these changes could have been responsible for the failure to observe an effect of perceptual completion on the accuracy of performance on different trials. The results from Experiment 3 also differed from the results of Experiment 1 , in that the impact of memory-test display congruity, which was based on a manipulation of perceptual completion, was confined to response speed on same trials. One explanation of this restriction can be developed from the differing nature of the component processes supporting same and different decisions. When, on same trials, the nature of the colored elements in a test display is congruent with how they were perceived in the memory display, a global comparison of the two displays will not generate any evidence for change, and there will be nothing to contradict a fast same response. However, when the 
memory and the test displays are incongruent, evidence for change will emerge from the global comparison of the displays, and additional processes will be required to ascertain whether the change involves task-relevant or task-irrelevant features. Because the manipulation of memory-test display congruity was irrelevant to the task decision, congruity would be expected to influence response speed, rather than response accuracy. The absence of a congruity effect on different trials has a relatively straightforward explanation on this account. The additional processes engaged to discover just how the two displays differ will involve examining only task-relevant features of the displays and so will not be influenced by the congruity of the memory and the test displays.

\section{GENERAL DISCUSSION}

When the participants in the present study were asked to remember the colors of the eight elements in a display, they took advantage of perceptual completion. It is assumed that performance was enhanced because perceptual completion allowed the number of items whose colors had to be remembered to be reduced from eight to four. In discussing this result, we argued that whatever new representation was created through perceptual completion, it did not replace the other forms of representation that were not contingent on completion and did not prevent these representations from continuing to support change detection. Thus, in the presence of perceptual completion, the participants continued to take advantage of other ways of organizing the displays perceptually, including perceiving the eight colored elements as separate entities. The participants continued to be sensitive to whether memory for the top or the bottom row of elements was probed, whether the identically colored elements were in corresponding ordinal positions in the two rows (i.e., were vertically aligned), and whether a change involved a color switch between elements from different bicolored groups or between elements from the same bicolored group.

What has emerged from the present experiments is a view of VSTM as a system capable of being supported simultaneously by multiple representations, each dealing with a different aspect of a display. The view that multiple representations can co-occur to support VSTM performance echoes the claims of others (e.g., Irwin \& Andrews, 1996; Lee \& Chun, 2001; Wheeler \& Treisman, 2002). However, these researchers contrasted object-based representations with a different form of representation than that being considered here. More specifically, they contrasted object-based representations with feature-based representations. In the present study, the participants were prevented from using feature-specific modules, such as might provide a list of feature values (i.e., colors) present in the memory display, because the changes that were made always involved two elements' exchanging their colors. This meant that the same feature values were present in the memory and the test displays.
Our claim is that the visual representations emerging from perceptual completion are object-based representations. This claim is made largely on the assumption that the products of perceptual completion are visual objects and anything that flows from completion will necessarily reflect this. But in what ways does perceptual completion reflect the nature of objects and the derivation of objectbased representations?

The algorithms underpinning visual perception appear to reflect two types of constraint: the first concerning some fundamental physical properties of objects, and the second concerning the very low probabilities associated with certain accidents of viewpoint (see, e.g., Rock, 1983). For example, objects are assumed to exist in a threedimensional (3-D) physical world independently of the presence of a human observer. They are assumed to continue to exist even when they become completely hidden to the observer by another object or when they can no longer be seen because the observer has turned to face away from them. As a result, in the absence of evidence to signal the removal of an object from potential view, the object will be expected to be seen when an occluding object is removed or when the observer changes position to a point and direction from which the object should be visible.

In addition to the constraints that flow from their physical properties, objects are also assumed to conform to a number of psychological constraints concerning accidents of viewpoint. For example, it is highly unlikely that two objects will have exactly the same surface shape in complementary form. It is even less likely that two such objects will be positioned in 3-D space so that their complementary boundaries perfectly abut each other directly in front of the observer. It is also unlikely that two such objects will be located at different distances from an observer but otherwise positioned so that their complementary contours are in perfect alignment along the observer's line of sight. The algorithms supporting visual perception appear not to accommodate such accidents of viewpoint. Instead, shared boundaries in the visual image are interpreted perceptually as belonging to only one object.

These various physical and psychological constraints on the nature and behavior of objects are especially apparent in perceptual completion. The shared border between two adjacent elements (i.e., two regions of uniform connectedness; see Palmer \& Rock, 1994) in a visual image is interpreted as belonging to only one object. A concomitant of this is that the two elements are perceived to arise from object surfaces located at different distances from the observer. Then, to avoid accommodating the accident of viewpoint just referred to, the object located at a more distant location is perceived to continue behind the occluder. Just how it is perceived to do so is determined by those of its features that remain visible to the observer.

On this account, perceptual completion occurs because visual perception acknowledges some of the fundamental physical properties of objects and is intolerant 
of accidents of viewpoint that might arise from the way in which objects are positioned in 3-D space relative to the observer. Considered in this way, perceptual completion is intimately linked with our understanding and perception of objects. Curiously, however, perceptual completion illustrates very well that perception can simultaneously remain faithful to the nature of an object and to the specifics of the viewpoint from which it is encountered. At the same time that it instills perception with an element of viewpoint independence, by representing the partially occluded object as if the occluder was not positioned between it and the viewer, perceptual completion does not prevent the occluding and occluded objects from being perceived from the observer's vantage point. There is, therefore, a curious mix of objectbased and viewer-specific representations. A similar mix has been observed in other studies concerned with visual object perception. For example, parts of an object that are currently hidden from the observer through self-occlusion (i.e., opaque objects necessarily hide some of their own aspects from the viewer) are thought to be included in a viewpoint-independent representation at the same time that a viewpoint-specific representation preserves details of only those aspects that are directly visible to the observer (see Lawson, 1999, for a recent review). It is the endeavor to represent the nature of objects independently of the specifics of a particular viewing position, a search for perceptual constancy, that qualifies perceptual completion and the representations it creates as being object based.

\section{REFERENCES}

Ceraso, J. (1985). Unit formation in perception and memory. In G. H. Bower (Ed.), The psychology of learning and motivation: Advances in research and theory (Vol. 19, pp. 179-210). New York: Academic Press.

Davis, G., \& Driver , J. (1997). Spreading of visual attention to modally versus amodally completed regions. Psychological Science, 8, 275281.

Ir win, D. E., \& Andrews, R. V. (1996). Integration and accumulation of information across saccadic eye movements. In T. Inui \& J. L. McClelland (Eds.), Attention and performance XVI: Information integration in perception and communication (pp. 125-155). Cambridge, MA: MIT Press.

Jiang, Y., Ol son, I. R., \& Chu n, M. M. (2000). Organization of visual short-term memory. Journal of Experimental Psychology: Learning, Memory, \& Cognition, 26, 683-702.

Kahneman, D., Tr eisman, A., \& Gibbs, B. J. (1992). The reviewing of object files: Object-specific integration of information. Cognitive Psychology, 24, 175-219.

Kanizsa, G. (1979). Organization in vision. New York: Praeger.

Kanizsa, G., \& Ger bino, W. (1982). Amodal completion: Seeing or thinking? In J. Beck (Ed.), Organization and representation in perception (pp. 167-190). Hillsdale, NJ: Erlbaum.

Kel 1 man, P. J., \& Shipl ey, T. F. (1991). A theory of visual interpolation in object perception. Cognitive Psychology, 23, 141-221.

Khurana, B. (1998). Visual structure and the integration of form and color information. Journal of Experimental Psychology: Human Perception \& Performance, 24, 1766-1785.

Lawson, R. (1999). Achieving visual object constancy across plane rotation and depth rotation. Acta Psychologica, 102, 221-245.
Lee, D., \& Chun, M. M. (2001). What are the units of visual short-term memory, objects or spatial locations? Perception \& Psychophysics, 63, 253-257.

Light, L. L., \& Ber ger, D. E. (1976). Are there long-term "literal copies" of visually presented words? Journal of Experimental Psychology: Human Learning \& Memory, 2, 654-662.

Luck, S. J., \& Vogel, E. K. (1997). The capacity of visual working memory for features and conjunctions. Nature, 390, 279-281.

Magnussen, S., \& Greenl ee, M. W. (1999). The psychophysics of perceptual memory. Psychological Research, 62, 81-92.

Pal mer, S. E., \& Rock, I. (1994). Rethinking perceptual organization: The role of uniform connectedness. Psychonomic Bulletin \& Review, 1, 29-55.

Pashl er, H. (1988). Familiarity and visual change detection. Perception \& Psychophysics, 44, 369-378.

Rau schenberger, R., \& Yant is, S. (2001). Masking unveils preamodal completion representation in visual search. Nature, 410, 369372.

Ren sin k, R. A., \& Enns, J. T. (1998). Early completion of occluded objects. Vision Research, 38, 2489-2505.

Rock, I. (1983). The logic of perception. Cambridge, MA: MIT Press. Schneider, W. X., Deu bel, H., \& Wesen ick, M.-B. (2000). Characterizing chunks in visual short-term memory: Not more than one feature per dimension? Behavioral \& Brain Sciences, 24, 144-145.

Sekul er, A. B., \& Pal mer, S. E. (1992). Perception of partly occluded objects: A microgenetic analysis. Journal of Experimental Psychology: General, 121, 95-111.

Sekul er, A. B., Pal mer, S. E., \& Flynn, C. (1994). Local and global processes in visual completion. Psychological Science, 5, 260-267.

Shor e, D. I., \& Enn s, J. T. (1997). Shape completion time depends on the size of the occluded region. Journal of Experimental Psychology: Human Perception \& Performance, 23, 980-998.

St efur ak, D. L., \& Boynt on, R. M. (1986). Independence of memory for categorically different colors and shapes. Perception \& Psychophysics, 39, 164-174.

Treisman, A. $\{$ M. $\}$ (1977). Focused attention in the perception and retrieval of multidimensional stimuli. Perception \& Psychophysics, 22, $1-11$.

Tr eisma n, A. M. (1998). Feature binding, attention and object perception. Philosophical Transactions of the Royal Society of London: Series $B, 353,1295-1306$.

Tr eisman, A. M., \& Gel ade, G. (1980). A feature integration theory of attention. Cognitive Psychology, 12, 97-136.

Tr eisman, A. M., Sykes, M., \& Gel a de, G. (1977). Selective attention and stimulus integration. In S. Dornic (Ed.), Attention and performance VI (pp. 333-361). Hillsdale, NJ: Erlbaum.

Vogel, E. K., Woodman, G. F., \& Lu ck, S. J. (2001). Storage of features, conjunctions, and objects in visual working memory. Journal of Experimental Psychology: Human Perception \& Performance, 27, 92-114.

Wal ker, P., \& Cut h bert, L. (1998). Remembering visual feature conjunctions: Visual memory for shape-color associations is objectbased. Visual Cognition, 5, 409-455.

Wheel er, M. E., \& Tr eisman, A. M. (2002). Binding in short-term visual memory. Journal of Experimental Psychology: General, 131, 48-64.

\section{NOTES}

1. The term accuracy is used to refer to all correct responses- that is, same decisions on same trials and different decisions on different trials. Although related to $d^{\prime}$, these accuracy scores allow performance on same and different trials to be examined separately.

2. We thank Nelson Cowan for drawing this issue to our attention.

(Manuscript received August 13, 2002; revision accepted for publication March 11, 2003.) 